\title{
Die Chemie-Abteilung der Ingenieurschule beider Basel
}

\author{
Men Barblan, Erich Flury*, Dieter Jahn und Karl Trefzer
}

Abstract. The essential features of the education of HTL chemists (equivalent to a B. Sc.) at Basle Engineering College are presented along with the aims of each subject. Some current problems are spotlighted.

\section{Zur Ingenieurschule beider Basel}

Die Ingenieurschule beider Basel in Muttenz ist, wie alle andern vom Bund anerkannten Ingenieurschulen, eine Höhere Technische Lehranstalt im Sinne von Art. 59 des Bundesgesetzes über die Berufsbildung vom 19. April 1978. Sie wird von den beiden Kantonen Basel-Stadt und Basel-Landschaft getragen und betrieben und besitzt das Statut einer selbständigen, öffentlich-rechtlichen Anstalt des Kantons Basel-Landschaft mit eigener Rechtspersönlichkeit. Die Oberaufsicht über die Schule übt der Regierungsrat des Kantons Basel-Landschaft aus.

Die Ingenieurschule beider Basel führt die Fachabteilungen Architektur, Bauingenieurwesen, Chemie, Elektrotechnik, Maschinenbau sowie Vermessungswesen. Ausserdem bietet die Ingenieurschule beider Basel die Nachdiplomstudien Energienutzungstechnik, Informatik und ab Herbst 1992 das Nachdiplomstudium Umwelt an. Ferner besteht die Möglichkeit, in der Abteilung Maschinenbau die Wahlrichtungen Betriebsingenieurwesen resp. Verfahrenstechnik und Apparatebau im Sinne eines Nachdiplomstudiums zu studieren.

Nach der 'Verordnung über Mindestvorschriften für die Anerkennung von Höheren Technischen Lehranstalten' [1] erfolgt die Aufnahme aufgrund einer Aufnahmeprüfung, zu der zugelassen wird,

*Korrespondenz: Dr. E. Flury

Ingenieurschule beider Basel

Abteilung Chemie

Gründenstrasse 40

$\mathrm{CH}-4132$ Muttenz
Chimia 46 (1992) 10-15 (c) Schweiz. Chemiker-Verband; ISSN 0009-4293 wer eine mindestens drei Jahre dauernde Berufslehre mit dem eidgenössischen Fähigkeitszeugnis abgeschlossen hat. Inhaber eines eidgenössisch anerkannten Maturitätszeugnisses müssen für den Eintritt in das erste Semester nur die Fachprüfung der Aufnahmeprüfung absolvieren. Zudem müssen sie eine der Fachrichtung verwandte praktische Tätigkeit von mindestens einem Jahr nachweisen. Absolventen der Chemie-Abteilung sind entspre- chend der Titelverordnung HTL [2] berechtigt, den Titel Chemiker HTL zu führen.

Gemäss dem Abkommen zwischen den Kantonen Basel-Stadt und BaselLandschaft [3] hat die Ingenieurschule beider Basel folgende Aufgaben: Sie

- vermittelt anwendungsbezogenes Ingenieurwissen;

- schult die Fähigkeit, schwierige Aufgaben aus dem Bereich der Technik, der Planung und der Gestaltung zu lösen;

- fördert die Persönlichkeitsbildung, das kritische Denken und die Urteilsfähigkeit;

- betreibt eine ganzheitliche Ausbildung, welche die Absolventinnen und Absolventen befähigt, Einflüsse ihrer Tätigkeit auf die Mitwelt zu erkennen und ihnen gegebenenfalls zu begegnen;

- sorgt durch das Angebot von geeigneten Veranstaltungen und Nachdiplomstudien für die ständige Fort- und Weiterbildung der in der Praxis stehenden Ingenieure und Ingenieurinnen;

- kann sich durch gezielte Forschungsarbeiten an der Weiterentwicklung und Aktualisierung technischen Wissens und der Grundlagenforschung beteiligen.

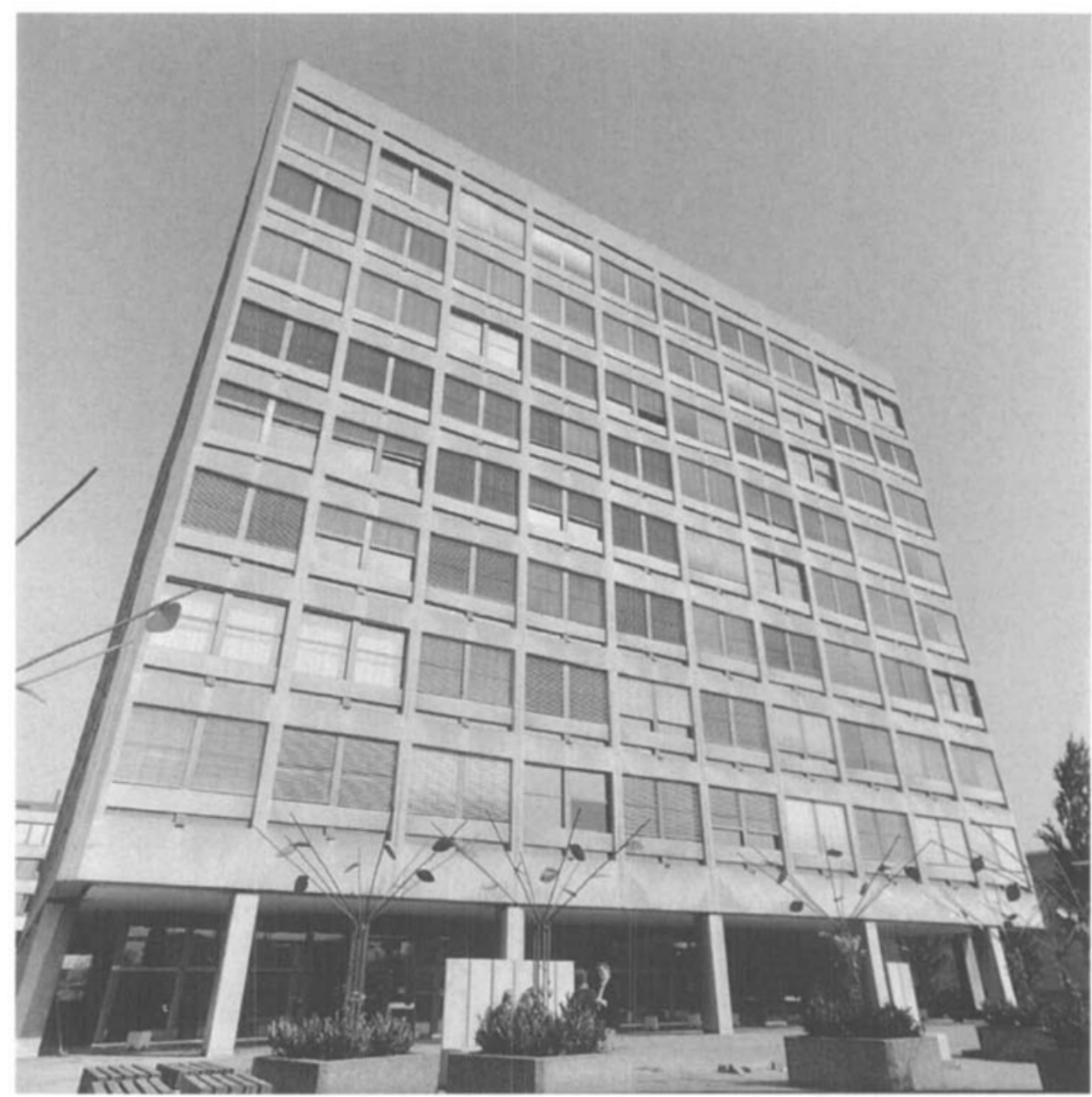

Abb. 1. An der Ingenieurschule beider Basel soll ... 


\section{Zur Chemieabteilung}

\subsection{Ausbildungsziel}

Die Chemieabteilung der Ingenieurschule beider Basel richtet ihre Ausbildungsziele vor allem nach den hauptsächlichen Einsatzbereichen ihrer Absolventinnen und Absolventen. Befragungen während mehrerer Jahre sowie gute Kontakte mit vielen Ehemaligen unserer Abteilung zeigen, dass sie überwiegend in chemischen Betrieben, Pilot-Anlagen, analytischen Laboratorien, Entwicklungslaboratorien sowie in Umweltschutz und Sicherheitsbereichen tätig sind. Eine beachtliche Anzahl wirkt aber auch mit Erfolg in Grenzgebieten wie z.B. im Ausbildungssektor, in der Applikation, in eher rechtlich orientierten Bereichen (Patentwesen, Ämter) oder technischer Verkaufsberatung, wo die sprach- und gesellschaftsorientierten Fächer von zusätzlicher Bedeutung sind.

Vor diesem Hintergrund entstand der letztmals 1986 gründlich revidierte und seither laufend neuen Erfordernissen angepasste Studienplan, dessen Stundentafel (s. Tab.) eine grobe Übersicht über die erteilten Fächer gibt.

\subsection{Kurze Charakterisierung der Fächer}

Während des derzeit sechs Semester dauernden Studiums haben sich die Studierenden der Chemie-Abteilung der Ingenieurschule beider Basel mit den nachfolgend beschriebenen Lerninhalten auseinanderzusetzen.

\subsubsection{Allgemeinbildende Fächer}

Mit den zeitlich zweifellos nur knapp dotierten sprach- und gesellschaftsorientierten Fächern soll ausser den allgemeinbildenden Gesichtspunkten die Tatsache berücksichtigt werden, dass auch die Chemikerinnen und Chemiker von Höheren Technischen Lehranstalten in einem Spannungsfeld zwischen Gesellschaft und beruflicher Tätigkeit stehen. So wird im Unterricht in Deutsch versucht, verbunden mit der Schulung der Sprache, Zugang zu den Werken der Literatur- und Geistesgeschichte zu finden und Probleme der Gegenwart einzubeziehen. Mit dem Unterricht in Englisch verfolgen wir das Ziel, unsere Studierenden so weit zu bringen, dass sie einfache englische Texte verstehen und korrekt schreiben können und dass sie in der Lage sind, Konversationen zu führen. Parallel dazu wird der Umgang mit englischer Fachliteratur geübt.

In der Rechtslehre sollten die Studierenden dazu gebracht werden, dass sie in der Lage sind, rechtliche Probleme in den richtigen rechtlichen Zusammenhang einzuordnen, einschlägige Rechtsquellen auf-

Tabelle. Stundentafel Abteilung Chemie

\begin{tabular}{|c|c|c|c|c|c|c|}
\hline \multirow[b]{2}{*}{ Semester } & \multicolumn{5}{|c|}{ Wochenstunden pro Semester } & \multirow[b]{2}{*}{6.} \\
\hline & 1. & 2. & 3. & 4. & 5. & \\
\hline \multicolumn{7}{|l|}{ Allgemeinbildende Fächer } \\
\hline Deutscn & 2 & 2 & 2 & 2 & - & - \\
\hline Englisch & 3 & 3 & 2 & 2 & - & - \\
\hline Rechtslehre & 2 & - & - & - & - & - \\
\hline \multicolumn{7}{|l|}{ Wirtschaftslehre einschliesslich } \\
\hline Wirtschafts-und Sozialgeschichte & - & 4 & - & - & - & - \\
\hline Unternehmung und Volkswirtschaft & - & - & 2 & - & - & - \\
\hline Betriebspsychologie & - & - & - & - & 2 & - \\
\hline \multicolumn{7}{|l|}{ Math. -naturw. Fächer } \\
\hline Ceometrie & 4 & - & - & - & - & - \\
\hline Mathematik & 5 & 3 & 4 & 3 & - & - \\
\hline Informatik & 1 & 2 & - & - & - & - \\
\hline Informatik in der Chemie & - & - & - & - & 2 & - \\
\hline Physik & - & 5 & 6 & 3 & - & - \\
\hline Physik-Praktikum & - & - & - & 4 & - & - \\
\hline \multicolumn{7}{|l|}{ Labor-orientierte Fächer } \\
\hline Allgemeine und anorganische Chemie & 6 & 2 & 2 & 2 & - & - \\
\hline Physikalische Chemie & 4 & - & - & - & 4 & 3 \\
\hline Analytische Chemie & - & 3 & 3 & 3 & - & - \\
\hline Organische Chemie & - & 4 & 4 & - & 4 & 4 \\
\hline Anorganisches und analytisches Labor & 9 & 9 & - & - & - & - \\
\hline Physikalisch-chemisches Labor & - & - & 4 & 4 & - & - \\
\hline Organisch-chemisches Labor & - & - & 8 & 8 & - & - \\
\hline Technisch-chemisches Labor & - & - & - & - & 8 & 8 \\
\hline \multicolumn{7}{|l|}{ Betriebsorientierte Fächer } \\
\hline Verfahrenstechnik & - & - & - & 2 & 4 & - \\
\hline Chemische Reaktionstechnik & - & - & - & - & - & 4 \\
\hline Seminar Technische Chemie & - & - & - & - & 2 & 3 \\
\hline Werkstoffe der chem. Technik & - & - & - & 2 & - & - \\
\hline Wärme- und Stoffaustausch & - & - & - & 2 & - & - \\
\hline Steuer- und Regeltechnik & - & - & - & - & - & 4 \\
\hline \multicolumn{7}{|l|}{ Verfahrensentwicklung, Anlagen- } \\
\hline planung und Betriebstechnik & - & - & - & - & - & 4 \\
\hline Chemie-Sicherheit-Umwelt & - & - & - & - & 4 & - \\
\hline $\begin{array}{l}\text { Verfahrens- und reaktions- } \\
\text { technisches Labor }\end{array}$ & - & - & - & - & 8 & 8 \\
\hline Total Stunden & 36 & 37 & 37 & 37 & 38 & 38 \\
\hline
\end{tabular}

zufinden und auf einen einfachen Sachverhalt anzuwenden.

Das Fach Wirtschaftslehre, einschliesslich Wirtschafts- und Sozialgeschichte, zeigt die wichtigsten sozialökonomischen Konzepte auf und soll die Studierenden dazu führen, dass sie gesamtwirtschaftliche Zusammenhänge mit Modellen mittleren Schwierigkeitsgrades analysieren können.

Im Fach Unternehmung und Volkswirtschaft werden Themen behandelt wie ökonomisch-politisch-rechtliche Rahmenbedingungen der industriellen Tätigkeit, wirtschaftlicher Erfolg und Lebensqualität und ausgewählte Sparten der chemischen Industrie mit ihren Märkten und ihrem gesellschaftlichen Umfeld.

Bekanntlich sind die schwierigsten und zermürbendsten Probleme im Berufsleben die zwischenmenschlichen. Es ist des- halb sicher sinnvoll, dass mit dem Fach Betriebspsychologie versucht wird, unsere Absolventinnen und Absolventen auf Situationen vorzubereiten, wie sie im betrieblichen Zusammenwirken von Menschen vorkommen können und wie auftretende Probleme erkannt und möglicherweise gelöst werden können.

\subsubsection{Mathematisch-naturwissenschaft-}

\section{liche Grundlagenfächer}

Mathematik und Physik als die Grundpfeiler von Naturwissenschaft und Technik bilden die Unterrichtsschwerpunkte in den unteren Semestern. Die Ausbildung in Mathematik führt von der einfachen Algebra zur Differential- und Integralrechung, zu Wahrscheinlichkeitsrechnung und Statistik. Sie soll unsere Absolventinnen und Absolventen befähigen, auch komplexere Zusammenhänge, wie sie den Alltag der 
Chemie-Ingenieurtechnik prägen, zu beschreiben und zu begreifen. Zusammen mit Geometrie, bei der Trigonometrie, Vektorrechnung und analytische Geometrie im Vordergrund stehen, sind für Mathematik im Studienplan insgesamt 19 Semesterwochenstunden, verteilt auf vier Semester, vorgesehen. Dieses Pensum wird von keinem anderen theoretischen Fach erreicht.

Die Ausbildung in Informatik soll die Studierenden in die Lage versetzen, einfache Programme unter Verwendung der Programmiersprache Turbo-Pascal selbst zu erstellen. Auch die Benutzung von Anwender-Software wird geübt, etwa im Zusammenhang mit Datenbanken, Tabellenkalkulation oder Textverarbeitung. Der Umgang mit dem Personalcomputer gehört ja längst zum Studienalltag, und sei es auch nur in Form des Verfassens von Laborberichten, der Erstellung von Graphiken oder der statistischen Auswertung von Versuchsreihen. Ein Seminar Informatik in der Chemie im letzten Studienjahr soll die speziellen Möglichkeiten der Informatik im Bereich der Chemie aufzeigen, von Laborautomation, Laborinformationssystemen, Qualitätssicherung bis zur Literaturrecherche. Dass sich hier die Möglichkeit von Studien 'vor Ort' in der Basler chemischen Industrie anbietet, ist naheliegend.

Der Unterricht in Physik versucht einerseits, den sozusagen allgemeinbildenden Aspekt dieses umfassenden Gebietes $\mathrm{zu}$ betonen, Begeisterung zu wecken für die Logik der Herleitung von Zusammenhängen und Gesetzen aufgrund von Experimenten und die Denkweise exakter $\mathrm{Na}$ turwissenschaft zu schulen. Andrerseits muss er vertieft vorbereiten auf sehr chemiespezifische Themen wie etwa die Spektroskopie. Physikunterricht für Chemiker darf und sollte sich unterscheiden von demjenigen des Ingenieurs. Besonders im halbtägigen Physikpraktikum lässt sich diesem Umstand Rechnung tragen.

\subsubsection{Labor-orientierte Fächer}

Die eigentlichen klassischen Teilgebiete der reinen und angewandten Chemie bilden natürlich das Kernstück auch unserer Chemikerausbildung. Auf sie kann nicht verzichtet werden, unabhängig davon, ob eine spätere Tätigkeit im Labor oder im Betrieb angestrebt wird. Wir haben sie gleichwohl 'labororientiert' genannt, um sie von den eher betriebs- und produktionsbezogenen Gebieten abzuheben. Bei allen hier genannten Fächern wird der theoretische Unterricht begleitet und unterstützt von entsprechenden Labor-Übungen.

Die allgemeine und anorganische Chemie möchte, mit Schwergewicht im ersten Semester, die allgemein-chemischen Themen wie die Natur der chemischen Bindung zur Sprache bringen, ohne dass die im Hochschulunterricht anzutreffende Tiefe und Ausführlichkeit angestrebt wird. Zusätzlich wird versucht, die Vielfalt der anorganischen Stoffchemie eher exemplarisch aufzuzeigen unter Betonung der Beziehungen zwischen stofflichem Aufbau und stofflichen Eigenschaften.

In der physikalischen Chemie sind weitere allgemeine und grundlegende Aspekte der Chemie zu behandeln, von der pragmatischen Anwendung von Formeln für die rechnerische Behandlung chemischer und elektrochemischer Gleichgewichte bis zur theoretischen Begründung thermodynamischer und kinetischer Grundgesetze. Seit einiger Zeit versuchen wir, der Berührungsangst mit diesem Fach dadurch zu begegnen, dass im ersten Semester ein Überblick mit Betonung auf den einfacheren Teilgebieten geboten wird, damit bereits zu Beginn des Studiums von wichtigen Grundbegriffen und Prinzipien eine gewisse Vorstellung besteht. Erst im fünften und sechsten Semester wird dann die Thematik erneut aufgegriffen und vertieft behandelt.

Die Ausbildung in analytischer Chemie erfolgt eigentlich nicht nur im Rahmen einer Vorlesung dieses Namens, sondern in starkem Masse auch fächerübergreifend. Die klassische qualitative anorganische Analytik wird bewusst vernachlässigt zugunsten einer ausführlichen Behandlung der Volumetrie und der Instrumentalanalytik mit den Schwerpunkten Chromatographie und Spektroskopie. Dabei werden die grundsätzlichen $\mathrm{Ge}$ sichtspunkte in den Vordergrund gestellt, während die speziellen Anwendungen, etwa Magnetische Kemresonanz zur Strukturaufklärung, eher im Rahmen anderer Fächer geübt werden.

Den Labor-Übungen im ersten Studienjahr (anorganisches und analytisches Labor) ist ein voller wöchentlicher Arbeitstag gewidmet. Die Betreuung der Studierenden erfolgt hier durch ein Team von Dozenten, welche den in Partnergruppen arbeitenden Studierenden Aufgaben stellen, die in der Regel sowohl anorganischpräparative als auch analytische Probleme zum Inhalt haben. Häufig wirken die Studierenden selbst mit bei der Formulierung von Problemstellungen. Das bringt Abwechslung, Anregung und Motivation auch für die Dozenten, und es ist eher die Ausnahme, dass Studierende eine Aufgabe haben, die im Vorjahr bereits von anderen Studierenden bearbeitet worden ist. Das Vermeiden von 'Routine-Versuchen' birgt allerdings die Gefahr in sich, dass einzelne Studierende bis zum Ende ihres Studiums gewisse Themen nicht selbst zu bearbeiten gezwungen sind. Grossen Wert legen wir darauf, dass die Studierenden mündlich und schriftlich über ihre Arbeiten berichten lernen und dass sie sich klar und adressatengerecht ausdrücken können.

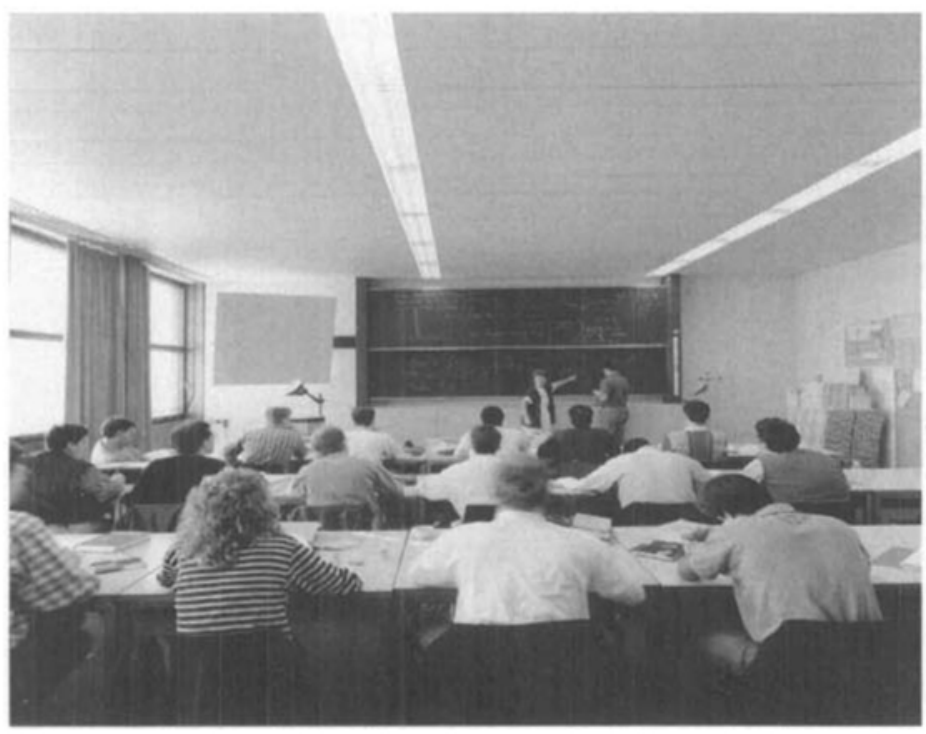

Abb. 2. ... der Frontalunterricht und ...

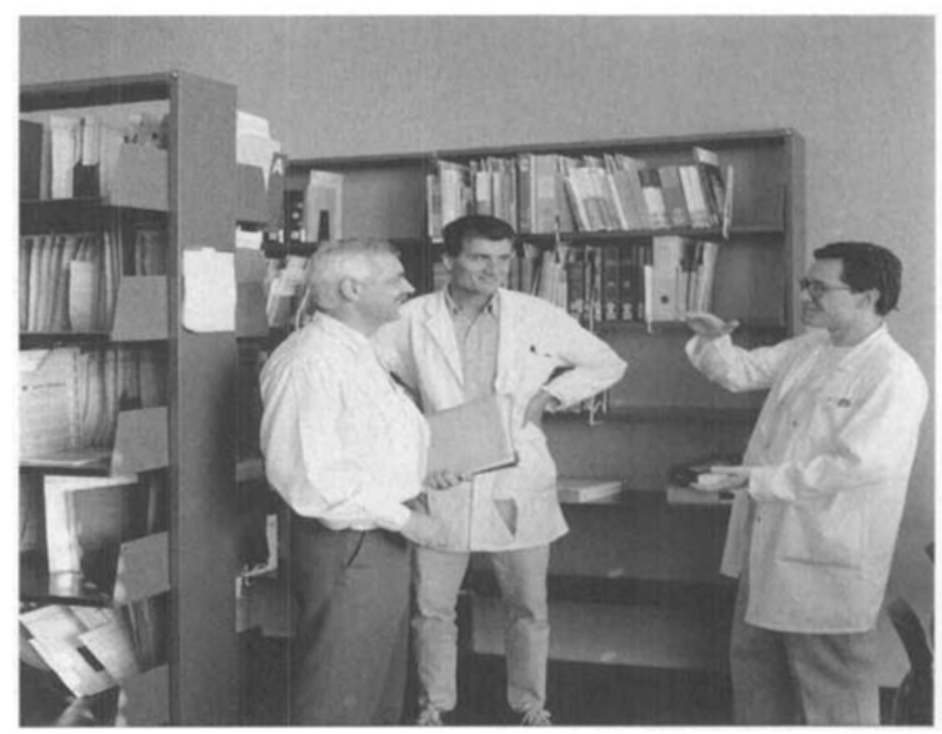

Abb. 3. ... das Lehrgespräch sowie ... 
Der erste Labortag ist für die Studienanfänger ein Tag grosser Unsicherheit. Wir konnten feststellen, dass sich unter der Anleitung der Dozentin für Betriebspsychologie diese kritische Phase der gegenseitigen Annäherung besser bewältigen lässt und dass das gegenseitige Vertrauen zunimmt, wenn Erwartungen und Befürchtungen artikuliert werden können.

Das halbtägige Physikalisch-chemische Labor des zweiten Studienjahres ist in starkem Masse auf instrumentelle Analytik im weitesten Sinne ausgerichtet und somit eine direkte Weiterführung des vorangehenden Praktikums.

In organischer Chemie, ergänzt durch das organisch-chemische Labor (ein wöchentlicher Arbeitstag im dritten und vierten Semester), wird das Lernziel verfolgt, dass die Studierenden die grundlegenden Reaktionen, Mechanismen, Anwendungen der Spektroskopie sowie Nomenklatur und Stoffeigenschaften wiedergeben und anwenden können. Im Zusammenhang mit präparativen und analytischen Aufgaben werden auch hier im Labor der Umgang mit der Fachliteratur sowie die mündliche und schriftliche Information geübt (Gruppeninformationen mittels Poster, Berichte über grössere Trenn- und Identifikationsaufgaben).

Das Technisch-chemische Labor des letzten Studienjahres wird von allen Chemiedozenten betreut und verfolgt das Ziel, die Studierenden in Form von Semesterarbeiten auf die selbständige Bearbeitung grösserer chemischer Probleme vorzubereiten, wie sie im späteren Beruf zu erwarten sind. Literaturbearbeitung und Konzepterarbeitung spielen hier eine besondere Rolle, und die Aufgaben werden meist nicht mehr Gruppen, sondern einzelnen Studierenden gestellt.

\subsubsection{Betriebsorientierte Fächer}

Die Durchführung chemischer Prozesse und Verfahren zur Herstellung eines chemischen Stoffes erfordern im Betriebsmassstabeinen weiterreichenden Aufwand als der Laborprozess. Ausgehend von der Tatsache, dass neben der Wirtschaftlichkeit des Verfahrens der Schutz der Menschen und der Umwelt prioritär ist, muss das ganze Herstellungsverfahren sehr sorgfältig bis in alle Einzelheiten analysiert, bearbeitet und optimiert werden. Wir bemühen uns, mit den folgenden Fächern unsere Studierenden in die Grundlagen des eher betriebsorientierten Bereiches einzuführen:

.Im Fach Chemische Reaktionstechnik wird der Ablauf chemischer Reaktionen analysiert und mittels kinetischer Modelle beschrieben. Zusammen mit dem mathematischen Reaktormodell kann anschliessend ein optimaler Reaktor ausgewählt,

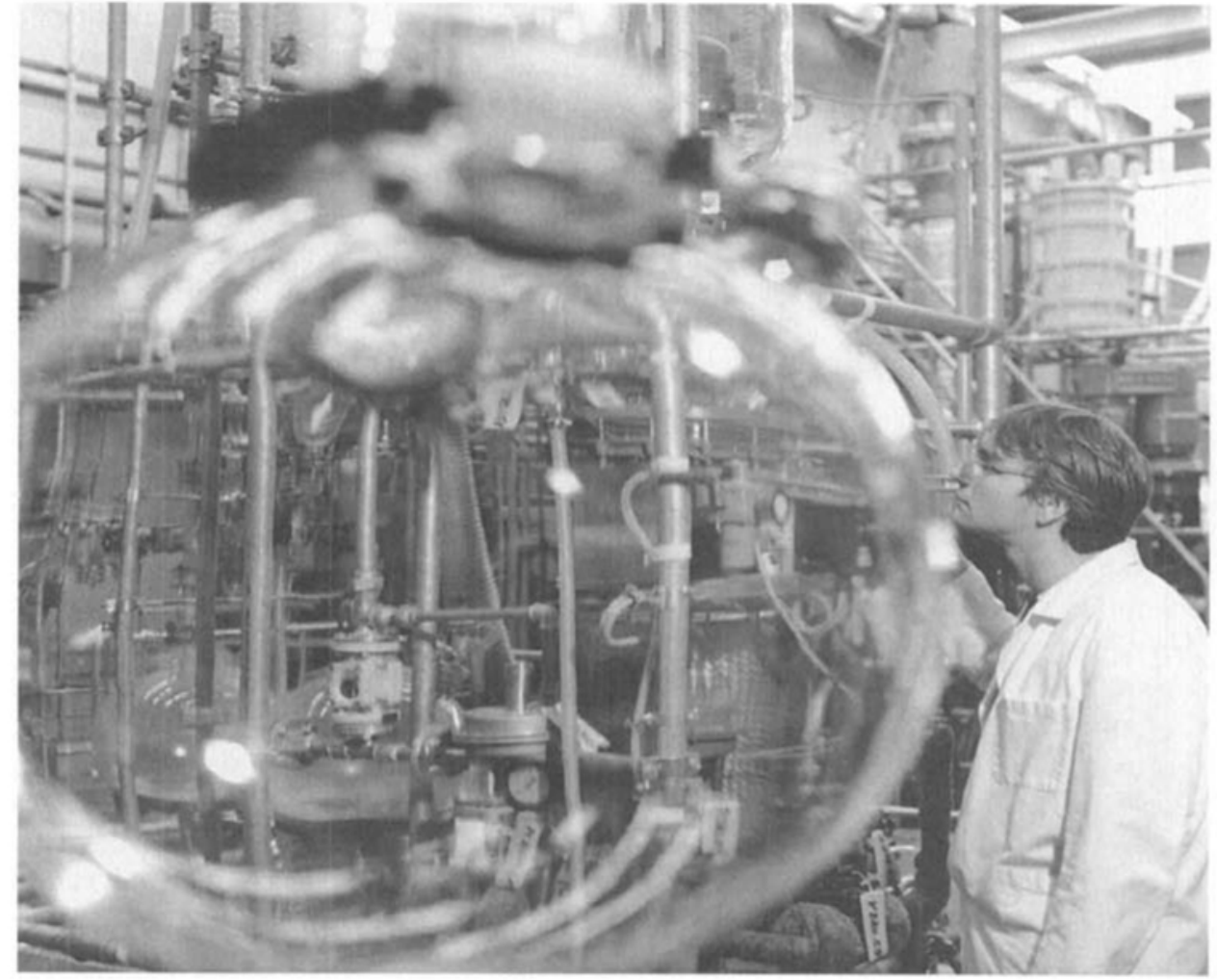

Abb. 4. ... eine transparente Beurteilung der Leistung...

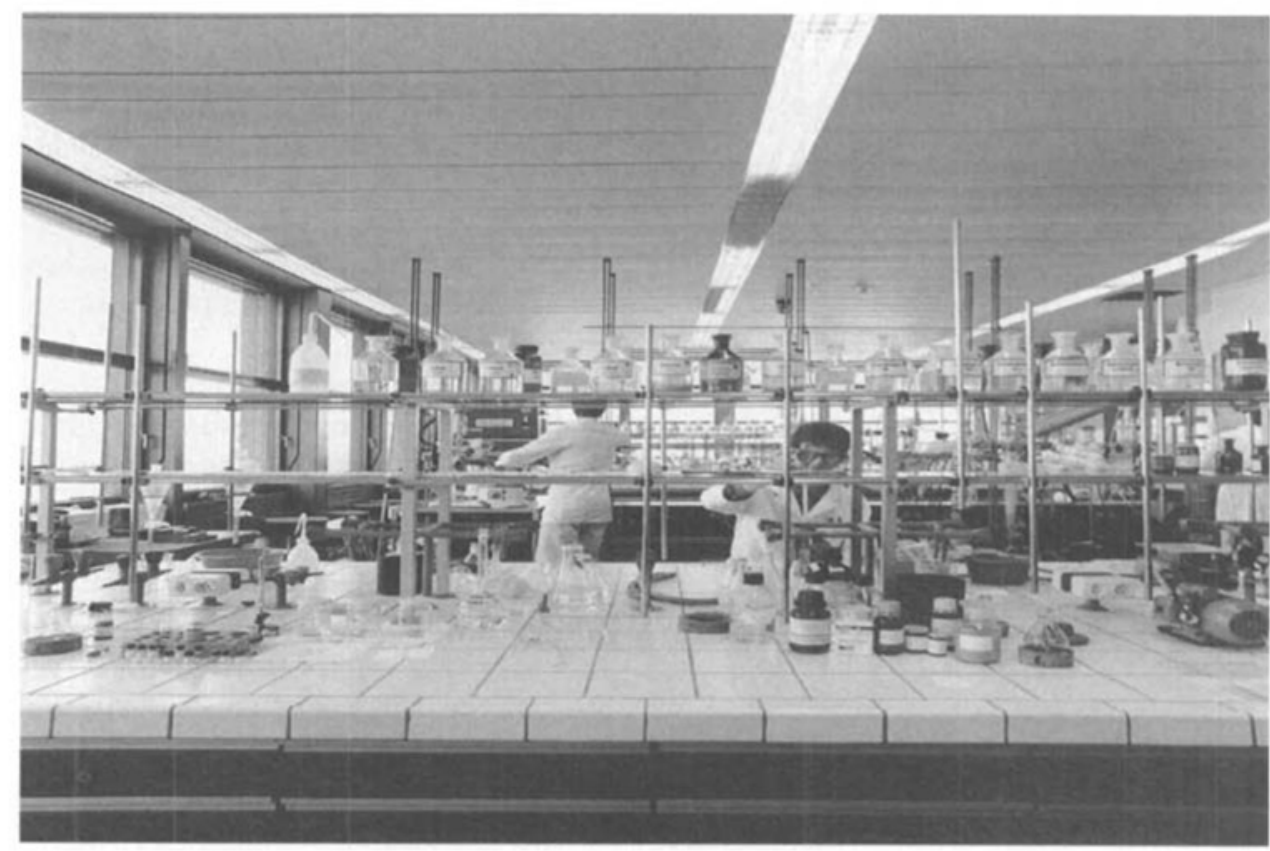

Abb. 5. ... die selbständige Arbeit im Labor und ...

dimensioniert und sein thermisches Verhalten vorausberechnet werden. Ergänzend dazu werden im Verfahrens- und Reaktionstechnischen Praktikum im sechsten Semester praktische Versuche zur Überprüfung der theoretischen Berechnungen durchgeführt. Gleichzeitig wird hier im Zusammenhang mit organischchemischen Reaktionen im Pilotmassstab der betriebsmässige Umgang mit Anlagen, Chemikalien und Lösungsmitteln erlernt.

Im Seminar Technische Chemie werden ausgewählte Kapitel aus der technischen Chemie wie Probleme der Herstellung organischer und anorganischer
Grundstoffe, Oberflächenschutz, Kolloide, Polymertechnologie, Optimierungsmethoden in der Chemie, Patentrecht usw. in seminaristischer Form behandelt. Zur Ergänzung werden Fachleute aus der Praxis beigezogen, und Kontakte mit der Industrie sollen die Veranstaltung illustrieren.

Die sicherheits- und umweltschutzrelevanten Eigenschaften von Chemikalien und deren Auswirkungen auf Mensch und Umwelt werden im Fach Chemie-Sicherheit-Umwelt behandelt und analysiert. Dabei erarbeitete Lösungen und Massnahmen werden in Anordnungen umgesetzt. Zusätzlich erfolgt eine Einführung in die Risikoanalyse. Ergänzend ist darauf 
hinzuweisen, dass in allen Laborübungen der Sicherheit und dem Umweltschutz neben dem (bereits in der Lehre erworbenen) korrekten experimentellen Verhalten, grosse Beachtung geschenkt wird. Auch auf die Bildung des Verantwortungsbewusstseins eines angehenden Chemikers und späteren Vorgesetzten wird besonderer Wert gelegt.

In Werkstoffe der chemischen Technik werden der Aufbau sowie die technologischen Eigenschaften von Werkstoffen und deren chemische und thermische Einsatzgrenzen behandelt.

Neben diesen Fächern mit vorwiegend chemischer und physikalisch-chemischer Ausrichtung benötigt man für die Durchführung chemischer Prozesse auch Apparate und Anlagen. Im Unterricht in Verfahrenstechnik werden neben der Funktionsweise der wichtigsten Apparate und Maschinen vor allem die mechanischen und thermischen Grundoperationen sowie die Fluiddynamik und das Fördern von Flüssigkeiten und Gasen behandelt. Als Ergänzung zum theoretischen Unterricht werden im verfahrenstechnischen Labor konkrete Aufgabenstellungen aus dem Gebiet der mechanischen und thermischen Verfahrenstechnik rechnerisch und praktisch gelöst.

Parallel zum ersten Teil des Verfahrenstechnik-Unterrichts im vierten Semester wird auch Wärme- und Stoffaustausch als Grundlage für alle thermischen Grundoperationen erteilt.

Die Verfahrens- und Anlagenplanung zeigt das Vorgehen bei der Weiterentwicklung eines Verfahrens vom Labor-

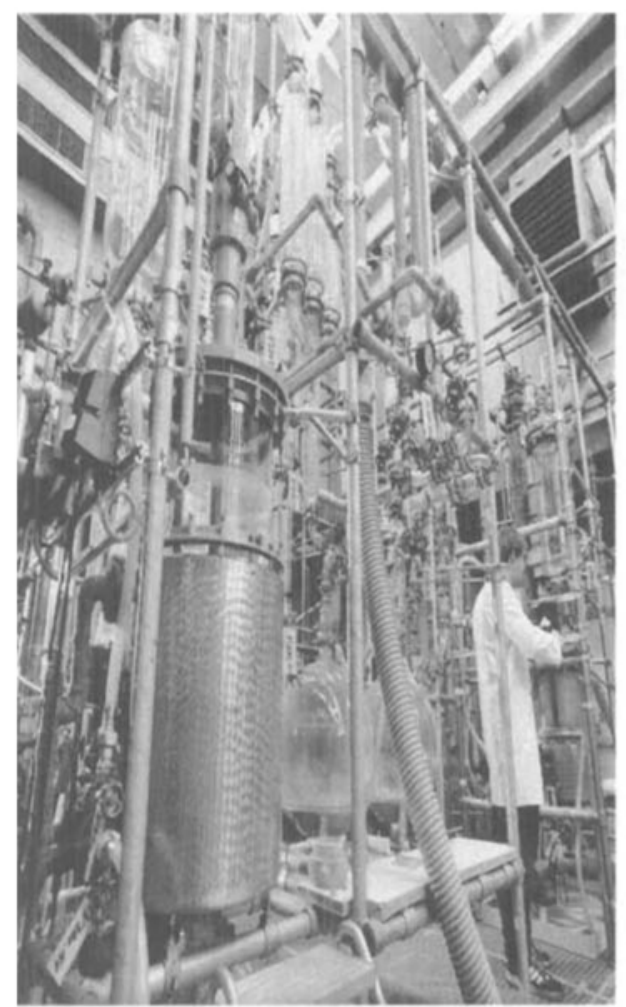

Abb. 6. ... im Betrieh fördern zum Betriebsmassstab, das Entwickeln von Verfahrens- und Anlagenkonzepten und 'scaling-up'-Methoden.

Da chemische Prozesse in verfahrenstechnischen Anlagen möglichst sicher und kontrolliert ablaufen sowie ein Produkt hoher und gleichmässiger Qualität liefern sollen, ist eine Anlagensteuerung unabdingbar. Die Auslegung und Projektierung von Steuer- und Regeleinrichtungen sowie das Einstellen von Regelparametern wird im Fach Steuer- und Regeltechnik geübt. Im Rahmen dieses Unterrichtes wird auch auf die Prozessleittechnik eingegangen.

Der Betriebschemiker muss sein Umfeld, bestehend aus den Funktionen Forschung, Entwicklung, Qualitätssicherung, Logistik, Einkauf, Marketing etc. mitsamt deren Aufgaben und Strukturen, kennen. Im Fach Betriebstechnik lernen die Studierenden diese Bereiche kennen und darin auftretende Probleme zu analysieren und zu lösen.

\subsection{Unterricht und Prüfungen}

Das zu Beginn dieses Artikels formulierte Ausbildungsziel kann in den derzeit verfügbaren drei Jahren nur durch eine straffe Unterrichtsführung und entsprechende Mitarbeit der Studierenden erreicht werden. Der Unterricht findet in der Regel in Form von vorlesungsartigen Lehrgesprächen im Klassenverband statt, worin auch Fragen, Diskussionen und Übungen ihren Platz haben.

Ein Blick auf die Stundentafel (s. Tab.) zeigt, dass unsere Studierenden ein Unterrichtspensum von 36 bis 38 Lektionen pro Woche zu bewältigen haben. Für die Bearbeitung (Vertiefung und Übungen) der im Unterricht vermittelten Lerninhalte sind pro Woche im Durchschnitt zusätzliche 15 bis 20 Arbeitsstunden einzusetzen. Bis auf wenige Ausnahmen wird in jedem Fach der Ausbildungsstand durch Klausuren geprüft. Die ersten drei und das fünfte Semester schliessen mit Noten ab, die für die Promotion in die folgenden Semester entscheidend sind.

Nach dem vierten Semester ist das Vordiplom zu bestehen, in dem Deutsch, Englisch, Allgemeine und Anorganische Chemie, Analytische Chemie und Physik geprüft werden. Das bestandene Vordiplom ist Voraussetzung zum Eintritt in das fünfte Semester.

Am Schluss des Studiums müssen die Absolventinnen und Absolventen mit einer insgesamt vier Wochen dauernden Diplomarbeit beweisen, dass sie die Fähigkeit besitzen, eine ihnen gestellte Aufgabe innerhalb der gesetzten Frist zu bearbeiten und die ihnen vermittelten Kenntnisse, die zugängliche Literatur und die zur Verfügung gestellten Geräte sinnvoll einzusetzen. Zusätzlich müssen sie in einer Diplomprüfung, bestehend aus schriftlichen oder mündlichen Prüfungen in $\mathrm{Or}$ ganischer Chemie, Physikalischer Chemie,Technischer Chemie, Verfahrens-und Reaktionstechnik sowie Steuer- und Regeltechnik, ihr theoretisches Wissen unter Beweis stellen.

Der umfangreiche Prüfungsstoff, der obligatorische Besuch des Unterrichtes und die starke Belastung während der Semester erfordern, dass sich die Studierenden auch während der im Vergleich zu den Hochschulen kurzen Ferien (insgesamt $c a$. zwölf Wochen, wovon sechs Wochen im Sommer als längste Ferieneinheit) auf die Vordiplom- und Diplomprüfungen vorbereiten.

\subsection{Mitsprache der Studierenden}

Partizipation an der Gestaltung des Alltages und der Zukunft der Schule ist nicht nur Gegenstand von Deklarationen in 'Papieren', die den Betrieb der Ingenieurschule beider Basel regeln, sondern auch Praxis unserer Chemieabteilung. So werden z.B. Lehrplanrevisionen in $\mathrm{Zu}$ sammenarbeit mit den Studierenden und mitumfangreichen Vernehmlassungen bei Ehemaligen der Abteilung durchgeführt. Institutionalisierte Strukturen wie Abteilungskonferenzen und Schulleitungskonferenzen mit Klassenvertretern erleichtern dabei einen geordneten Ablauf der internen Meinungsbildung. Oft resultiert bei diesem breit durchgeführten Prozedere eine Vielzahl von teilweise gegensätzlichen Meinungsvarianten, die vom Leitungsteam der Abteilung unter allfälligem Miteinbezug Betroffener sorgfältig bearbeitet und einer möglichst optimalen Lösung zugeführt werden. Seit dem Bestehen unserer Abteilung (Eröffnung 1971) hat sich dieses Vorgehen auch aus der Sicht der Direktion unserer Schule und der unmittelbaren Aufsichtsbehörde, des Technikumsrates, sehr bewährt.

\subsection{Externe Kontakte}

Eine Höhere Technische Lehranstalt ist eine Abschlussschule, d.h. eine Schule, die ihre Absolventinnen und Absolventen mit einem ausübbaren Beruf (Chemikerin HTL/Chemiker HTL) entlässt. Sie muss sich deshalb an den Erfordernissen der Praxis orientieren und im Fall unserer Chemie-Abteilung zu geeigneten und interessierten Vertretern aus der Wirtschaft und der Verwaltung gute Kontakte pflegen. So beleben und illustrieren Exkursionen in die chemische Industrie, vor allem in den oberen Semestern der Ausbildung, den Unterricht und geben den Studierenden Perspektiven für ihre spätere berufliche Tätigkeit. Bei vielen Fragestellungen gelangen wir an Ehemalige, die uns aus 
der Sicht ihrer oft anspruchsvollen Funktionen gerne beratend oder auch im Unterricht mit Vorträgen zur Seite stehen. Aber auch der Besuch von Kursen, Tagungen von Fachverbänden oder grösseren Weiterbildungsanlässen bringt die Dozentenschaft neben dem Erwerb zeitgemässen Fachwissens zu Kontakten mit Vertretern von Wissenschaft und Technik. Weitere sehr wichtige Kontakte mit der Praxis gewährleistet auch die beträchtliche Anzahl an nebenamtlichen Dozenten, die meist nur wenige Wochenstunden in Spezialgebieten unterrichten.

Zu erwähnen ist ferner der 1991 schon zum fünften Mal von der Chemie-Abteilung unserer Schule in Zusammenarbeit mit der Schweizerischen Akademie der Technischen Wissenschaften (SATW) und der DECHEMA organisierte Kurs über Formulierungstechnik

\subsection{Derzeitige Probleme}

\subsubsection{Andrang von Studierenden}

Die Chemie-Abteilung unserer Schule kann pro Jahr maximal 30 Studierende aufnehmen (Laborplätze). Die 'Statistik der Abteilung Chemie' (Fig.) zeigt, dass seit 1986 immer mehr als 30 Kandidatinnen und Kandidaten die Aufnahmeprüfung bestanden haben. Dieser Sachverhalt hat zur Folge, dass in den vergangenen fünf Jahren einer zunehmenden Anzahl erfolgreicher Absolventinnen und Absolventen entweder ein anderer Studienort mit noch freier Aufnahmekapazität (z.B. Winterthur mit zwei Klassenzügen) oder eine zeitliche Verschiebung ihres Studienbeginns zugemutet werden musste.

Da wir in der Regel im Klassenverband unterrichten und das Lehrgespräch pflegen (den Voraussetzungen der Absolventinnen und Absolventen angepasst und zudem vom BIGA [1] gefordert) führt die erwähnte Maximalbelegung einer Klasse in einigen Fächern zu pädagogisch unbefriedigenden Situationen. Die beiden Sachverhalte zwingen deshalb zur Auseinandersetzung mit der Frage, ob die ChemieAbteilung unserer Schule einen zweiten Klassenzug führen soll. Beim schon seit einiger Zeit herrschenden Raummange und der finanziellen Situation der Trägerkantone Basel-Landschaft und BaselStadt, sowie angesichts der Tatsache, dass die Chemie-Abteilung der Ingenieurschule Winterthur jährlich 10-15 erfolgreiche Absolventinnen und Absolventen unserer Aufnahmeprüfung aufnehmen könnte, ist aber jede Bemühung zur Schaffung von zwei Klassenzügen fragwürdig.

\subsubsection{Studienreform}

Die kritische Durchsicht des Lehrplanes sowie Gespräche mit Studierenden

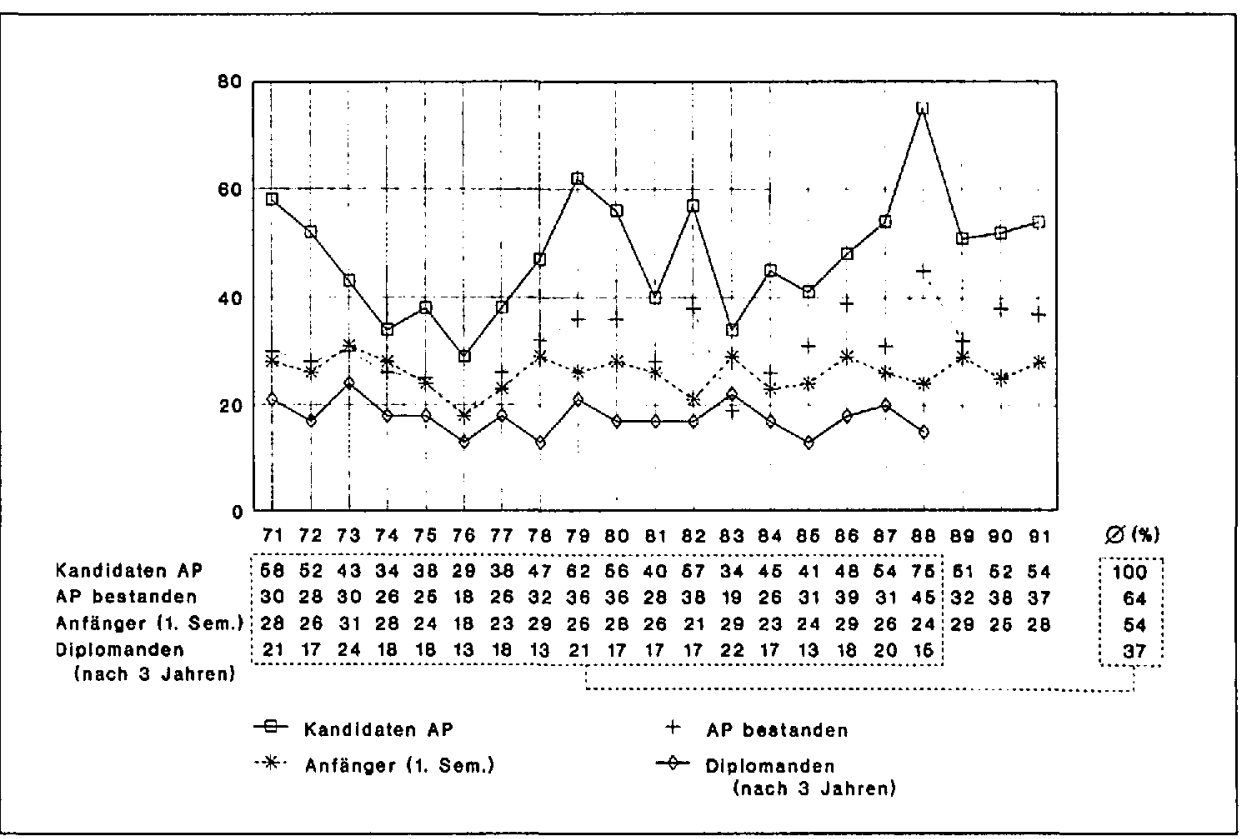

Fig. Statistik der Abteilung Chemie (AP = Aufnahmeprifung)

oder Ehemaligen führen sehr oft zur Schlussfolgerung, dass die Studierenden nicht in der Lage sind, die vermittelten Lerninhalte über ein als nur 'befriedigend" zu bezeichnendes Mass hinaus zu assimilieren.

Zweifellos liegt eine wesentliche Ursache darin, dass die rasanten Entwicklungen in Wissenschaft und Technik auch in den Höheren Technischen Lehranstalten zuzuordnenden Gebieten ihre Berücksichtigungen fordern und dass wenig kompensatorische Abstriche bei den Grundlagen möglich sind. Ein weiterer Grund ist in der notwendigen aber zeitaufwendigen $\mathrm{Be}$ schäftigung mit dem Hilfsmittel Computer zu finden.

$\mathrm{Da}$ von unseren Absolventinnen und Absolventen ein gewisses Mass an Kenntnissen im Umgang mit dem Computer erwartet wird und da auf die zeitgemässen wissenschaftlichen und technischen Fachkenntnisse der an einer HTL ausgebildeten Chemikerinnen und Chemiker auch nicht verzichtet werden kann, sind die Studierenden gezwungen, entweder übermässigen Zusatzaufwand zu leisten oder an der Assimilationsqualität der vermittelten Lerninhalte Abstriche zu machen. Daraus ergeben sich sowohl für die Lernenden als auch die Lehrenden recht unbefriedigende Situationen.

Vor diesem Hintergrund muss deshalb, obwohl mit der chemie-pädagogischen Zielsetzung im Widerspruch stehend, gelegentlich bildverzerrende Nachsicht bei der Qualifikation mangelhafter Leistungen in der Theorie und in den Praktika geuibt werden.

An der Ingenieurschule beider Basel finden derzeit auf allen Ebenen Diskussionen über Studienreform-Varianten statt, die der unbefriedigenden Assimilationsqualität der vermittelten Lerninhalte be- gegnen und die vieldiskutierte europäische oder internationale Anerkennung der Diplome von Höheren Technischen Lehranstalten der Schweiz einschliessen sollen. Allerdings scheinen derzeit die Forderungen derZukunft den Finanzperspektiven zuwiderzulaufen. Von allen Beteiligten ist zur Lösung dieser Probleme ein hohes Mass an Optimierungsphantasie sowie auch an Einsicht und Engagement gefordert.

\subsubsection{Weitere Probleme}

Zu erwähnen und zu diskutieren wären die Sicherheit und die Entsorgung von Chemieabfällen in den Praktika sowie damit verbundene infrastrukturelle Probleme, die Berücksichtigung modemer Entwicklungen im Unterricht und in den Laboratorien, die Schaffung von Wahlrichtungen und Wahlfächern usw. Da das Ziel dieses Artikels aber als Portrait und weniger als Durchleuchtung vorgegeben war, wird hier auf die Diskussion weiterer Probleme verzichtet.

Frau A. Brandenberg danken wir für ihre Mitwirkung sowohl als Ehemalige als auch als Assistentin der Chemie-Abreilung.

[1] Verordnung über die Mindestvorschriften für die Anerkennung von Höheren Technischen Lehranstalten vom 8. Oktober 1980 (Eidg. Volkswirtschaftsdepartement).

[2] Verordnung über bestimmte Titel für Absolventen von Höheren Technischen Lehranstalten vom 8. Oktober 1980 (Eidg. Volkswirtschaftsdepartement).

[3] Abkommen zwischen den Kantonen BaselStadt und Basel-Landschaft zum Betrieb der Ingenieurschule beider Basel (HTL) in Muttenz vom 11./12. September 1989 (Landeskanzlei Kt. Bassel-Landschaft).

Fotos: Jehle, Pratteln 